\title{
THE
}

\section{Emotional reactivity monitoring using electrodermal activity analysis in individuals with suicidal behaviors}

Amir Mohammad Amiri

University of Rhode Island

Mohammadreza Abtahi

University of Rhode Island

Anna Rabasco

Michael Armey

Kunal Mankodiya
Eullow this and additignal works at: https://digitalcommons.uri.edu/ele_facpubs
lnuversitv

The University of Rhode Island Faculty have made this article openly available.

Please let us know how Open Access to this research benefits you.

This is a pre-publication author manuscript of the final, published article.

Terms of Use

This article is made available under the terms and conditions applicable towards Open Access

Policy Articles, as set forth in our Terms of Use.

\section{Citation/Publisher Attribution}

Amiri, A. M., Abtahi, M., Rabasco, A., Armey, M., \& Mankodiya, K. (2016, March 20-23). Emotional reactivity monitoring using electrodermal activity analysis in individuals with suicidal behaviors. 2016 10th International Symposium on Medical Information and Communication Technology (ISMICT), Worcester, MA. doi: 10.1109/ISMICT.2016.7498896

Available at: http://dx.doi.org/10.1109/ISMICT.2016.7498896

This Article is brought to you for free and open access by the Department of Electrical, Computer, and Biomedical Engineering at DigitalCommons@URI. It has been accepted for inclusion in Department of Electrical, Computer, and Biomedical Engineering Faculty Publications by an authorized administrator of DigitalCommons@URI. For more information, please contact digitalcommons-group@uri.edu. 


\title{
Emotional Reactivity Monitoring Using Electrodermal Activity Analysis In Individuals With Suicidal Behaviors
}

\author{
Amir Mohammad Amiri, Mohammadreza Abtahi, Anna Rabasco, Michael Armey, \\ Kunal Mankodiya, Member, IEEE
}

\begin{abstract}
Suicide, considered as one of the most leading causes of death, has not given enough and appropriate attention in order to reduce its rate such that the humans in all over the world deserve it. The problem addressed in this paper is analysis of the relation between an extra stimulus and physiological data's responses. In order to record the physiological data set from multiple subjects over many weeks, we used an acoustic startle during a Paced Auditory Serial Addition Task (PASAT) test that spontaneously leads subjects to real emotional reactivity, without any deliberate laboratory setting. Crucially, we show that, by inducing anxiety during the test, changes appear in Electrodermal activity, Electrocardiogram, Heart Rate and Respiration Rate. A wide range of physiological features from various analysis domains, including modeling, time/frequency analysis, an algorithm and etc., is proposed in order to find the best emotional reactivity feature to correlate them with emotional states which can be considered as a suicide factor. More specifically, this paper is focused on the EDA data analysis. Experimental results highlight that all cited techniques perform well and we achieved a high resolution of tonic and phasic components which allow us to measure the latency, onsets and amplitudes of EDA responses to a stimulus. This paper follows the association of recommendations for advancement of health care instruments.
\end{abstract}

Index Terms-Suicide Behavior, Emotional Reactivity, Electrodermal Activity, Electrocardiogram, Heart rate, Respiration rate.

\section{INTRODUCTION}

$\mathbf{S}$ UICIDAL behavior is a tragic phenomenon for mental health issue and may be imminence for a society if not prevented. It remains a serious public health problem and reducing suicide rate is a government priority. In a report released by American Foundation for Suicide Prevention (AFSP), it is mentioned that suicide accounts is the third after cancer and heart disease, for more years of life lost than any other causes of death [1].

According to a 2014 report from Disease Control and Prevention (CDC), there has been no increase in the rate of the top ten leading causes of death in the United States, except the tenth factor suicide. In 2013, suicide stood as the tenth leading cause of death in the United States with more than 41,000 suicides reported. In other words, someone died by suicide every 12.8 minutes [2].

Amir Mohammad Amiri, Mohammadreza Abtahi and Kunal Mankodiya are with University of Rhode Island, Kingston, USA. \{amiri, mabtahi, kunalm\}@ele.uri.edu

Michael Armey is with University of Brown, USA.
The definition of suicide by CDC, is death caused by selfdirected injurious behavior with an intent to die as a result of that behavior [3]. Suicidal risk assessment divides into three groups of participants: i) suicide attempt (SA): an attempting act that the person refers to self-inflicted harm where death does not occur, but the intention of the person was to cause a fatal outcome, ii) suicidal ideation (SI): thinking about, considering, or planning to suicide, iii) non-suicidal self-injury (NSSI): the deliberate, self-inflicted destruction of body tissue with no suicidal intent or other culturally sanctioned purposes which results in immediate damage [4].

Suffering from various types of mental health problems, there are a number of people with personality disorders and terrible life events, but never considered committing suicidal act or self injury. Risk factors for suicide are characteristics or conditions that increase the chance that a person may try to take their life that are often confused with warning signs of suicide. Health, environmental and historical circumstances like mental health conditions, chronic illness, psychiatric disorders, stressful life events, trauma, heritability and family history, are three major factors that may contribute to a person's risk of suicide [5].

Although there are different factors bringing up the idea of suicide, but all the patients need proper treatment which is very challenging. The most common treatments are: Hospitalization, Outpatient treatment, and medication or other modalities [6]. In hospitalization, the patients should be supervised and secured in the hospital. Patients showing the tendency to harm themselves or other people, or patients who have access to some dangerous tools are the best fits for hospitalization treatment [7]. In contrast to hospitalization, the patients stay at their own place in outpatient treatment having some scheduled visits with doctors for receiving their treatments [8]. Medication treatment, which comes from the name, means prescribing antidepressant medicines as a treatment for the patients, but it should be done very carefully since some of these medicines may act reversely and increase the risk of suicide [9].

The study of suicidal risk phenomena portrayed by humans during social interactions, requires rich sets of labeled data with stimulated situations occurring in daily-life/laboratory. Such datasets enable researchers to have a better understanding of the correlations that may exist between suicide behavior and physiological data e.g., Electrodermal Activity (EDA), Electrocardiogram (ECG), Electromyography (EMG) and Electroencephalogram (EEG) and respiration signals. By 


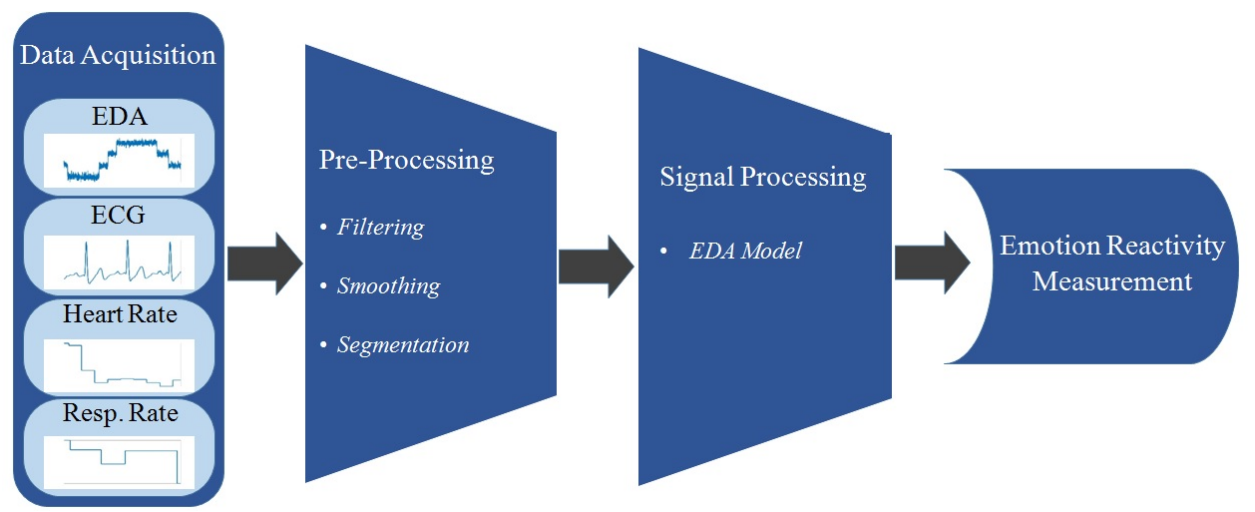

Fig. 1. Schematic flow-work of Emotion Reactivity Measurement.

developing systems that utilize extracted features from sensors (measured signals), this identification can be achieved. And for being used in suicidal risk assessment computer-aided analysis or an android device to predict the suicide acts, these systems need to have well enough performance.

In this paper, we have multimodal recordings that include psychophysiological data such as ECG, EDA, Heart Rate and Respiration Rate signals and the changes in these signals as the response to the stimulus are recognizable. In this study, more specifically we analyze the EDA signal which help to recognize the suicide behaviors based on evaluation of responses to an extra stimulus. The results obtained through the comparison of stimulated with baseline.

\section{RELATED WORK}

Some studies have been performed with the goal of assessing the suicidal risk providing tools for practical suicide signs detection and improving the prediction or diagnostic accuracy of physicians in small practice settings.

In a report by Schumm et al. [10], the effect of quasistationary movements on the EDA after a startling event has been investigated and evaluated. The goal of this study was to expand the knowledge about EDA in real life applications. They could record EDA and finger movements simultaneously by designing a comfortable body-worn measurement device. This study recruited five subjects to walk at different speeds listening to acoustic startles. Using crosscorrelograms and cumulative frequency plots, the EDA response to the startle for different walking speeds was analyzed.

In another report by Wang et al. [11], atypical electrodermal and cardiovascular response patterns in psychopathic individuals is mentioned that would be thought as fearless and disinhibition biological indicators. By using a count-down task in 843 children, this study investigated the relationship between these autonomic response patterns and psychopathic traits. While participants reacting to $105 \mathrm{~dB}$ signaled or unsignaled white-noise bursts, Heart rate (HR) and non-specific skin conductance responses (NS-SCRs) were recorded. It is found that both fewer NS-SCR and larger HR acceleration are strongly associated with psychopathic traits during anticipation of signaled white-noise bursts, by using multilevel regression models. Two divergent patterns appeared for HR and SCR:
(1) larger HR acceleration was specific to the callousnessdisinhibition factor of psychopathic traits while reduced NSSCR was only associated with the manipulative-deceitfulness factor; (2) the negative association between the manipulativedeceitfulness factor and NS-SCR was only found in boys but not in girls. Those findings replicated what has been found in psychopathic adults, suggesting that autonomic deficits present in children at risk may predispose them to later psychopathy.

\section{DATA ACQUISITION}

\section{A. Participants}

Data have been recorded from four patients aged 25-35 with current suicidal ideation or a recent suicide attempt amongst the patients in Butler Hospital, Providence, RI, USA.

\section{B. Protocol}

Participants completed a brief series of behavioral and laboratory assessments during their inpatient stay in a quiet room dedicated to physiological assessment. The Paced Auditory Serial Addition Task Computer Version (PASAT-C) 47 is a behavioral manipulation designed to induce frustration and emotional upset in the laboratory, which has been characterized as a behavioral measure of emotion reactivity. The PASAT-C requires participants to add numbers within a set time period. As the task progresses, participants are required to respond more rapidly, producing stress and discomfort. In this study, three phases of PASAT (PASAT-5,3,1) was selected with the time sections of 5,3 and 1 seconds respectively (see figure 2). Participants complete a subset of the PANAS positive and negative emotion items (6 items total) as well as a six-item measure of distress tolerance. During the last phase, participants were instructed that they could quit whenever they like, but were encouraged to continue for as long as they could. Participants first underwent 15 minutes of baseline resting physiological assessment followed by an acoustic startle assessment, administered using a standard white-noise burst $(500 \mathrm{~ms}, 120 \mathrm{~dB})$ presented through an earpiece placed in the participants left ear. 


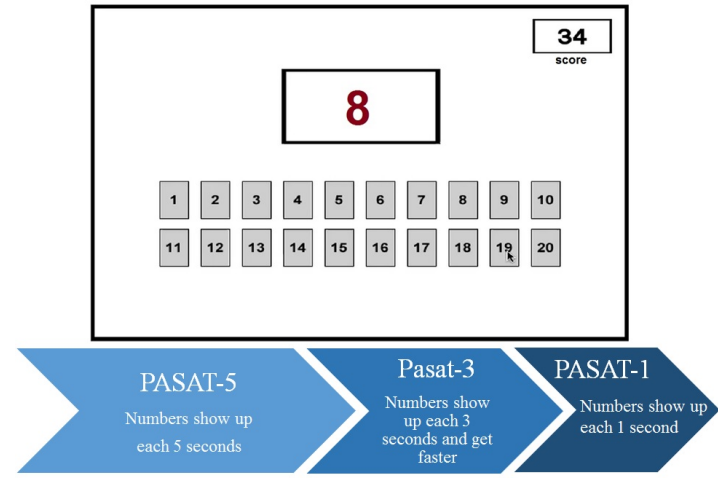

Fig. 2. A screenshot of PASAT test along with time duration of each phase.

\section{Psychophysiological Data}

SC, Pulse and Respiration were measured using a single fingertip sensor attached with Velcro to the participants nondominant thumb, and a chest strap around the participants chest, aligned with the sternum respectively. Physiological arousal and reactivity, including SC, have been monitored continuously throughout laboratory procedures of baseline relaxation (i.e. startle blink), PASAT-C, and recovery/relaxation period. Data was acquired using the Biopac MP150 interfaced to a computer. Signal and noise analog data have been monitored on the computer and digitized data was stored for analysis with the AcqKnowledge Specialized Analysis Package, which automates higher-level analysis.

\section{Methods}

In this section, extracting features for analyzing data after administration of the startle is described according to the actual step performed in pipeline by EDA data.

The skin's electrical phenomena that make skin as a better conductor of electricity when a stimulus occurs, the skin conductance response call as Electrodermal Response. [12]. The electrical properties can be viewed through the interpretation of skin conductance and/or skin potential which requires a knowledge and understanding of tissues structure in the skin surface. The tissue of sweat gland activity contains a simple tube made up of the single/double layer of epithelial cells [13].

SC can be measured by applying a low constant voltage and normally an $8 \mathrm{~mm}$ diameter silver chloride electrode. The activity of sweat glands is triggered by post-ganglionic sudomotor fibers which plays a major role in thermo-regulation and keeping the skin flexible for sensory discrimination. That is also a concomitant of the orienting response and more general of emotional arousal. Clinical application encompasses a variety of fields, such as the assessment of emotion reactivity, pain, schizophrenia or peripheral neuropathy [14].

Skin conductance divides into two major components [15]:

- Skin Conductance Level (SCL) provides a tonic which is defined as the baseline level of skin conductance in the absence of any particular discrete environmental event and slowly habituating measure of arousal. SCL is different for each person and with typically tonic levels ranging from $10-50 \mu S$. In particular, tonic skin conductance levels are related to Autonomic Nervous System (ANS) regulation which depends on his or her psychological state [16].

- Skin conductance response (SCR) provides a phasic when events take place (startle in this study), moment-bymoment measure of arousal reflecting stimulus (sights, sounds, smells, etc.) specific responses or non-specific orienting. SCR increases with the skin conductance which may last 1-20 seconds occurred by a return to the tonic or baseline level of skin conductance. These phasic changes are called as GSRs and generally, a spontaneous GSR is between 1-3 per minute. Event-related SCRs are traditionally analyzed by the amplitude comparison of individual peaks against a pre-stimulus baseline [17].

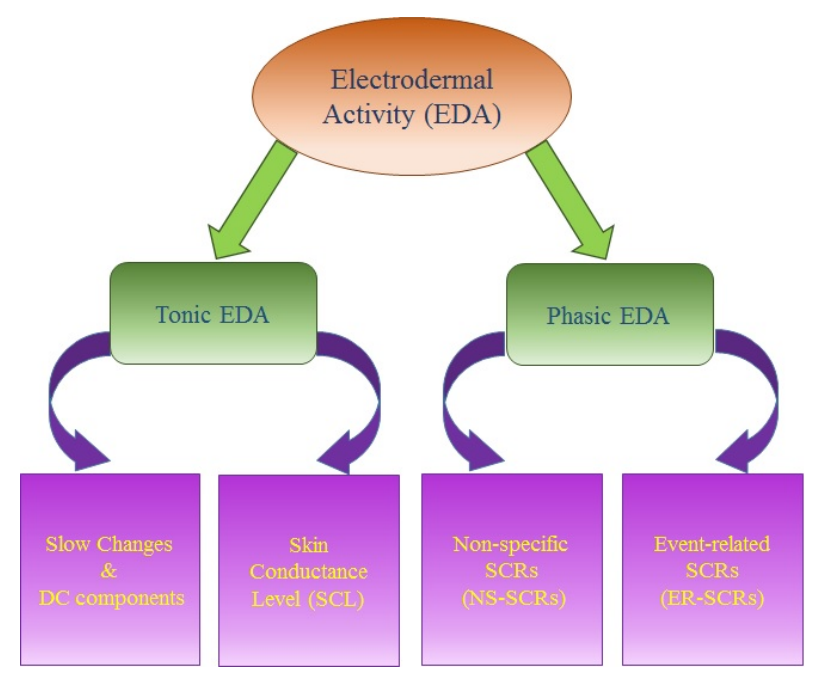

Fig. 3. An illustration of Electrodermal components.

A number of physiological studies show the SCR is caused by discrete bursts of the sudomotor nerves that control the sweat glands [18].

It is shown that the skin conductance time-series can be given by a differential equation:

$$
a \frac{d^{2} G}{d t^{2}}+b \frac{d G}{d t}+G(t)=S(t)
$$

where $a=\tau_{0} \tau_{1}, b=\tau_{0}+\tau_{1}$ and $\tau_{0}, \tau_{1}$ are time-constants

The skin conductance is defined as $G(t)$ and the driver function of the differential equation is $S(t)$ which can be considered to show the sudomotor nerve activity. The decaying tails of the SCRs and the rise time in response to a peak in the driver are derived by larger and shorter time-constant $\left(\tau_{0}\right)$ respectively.

It is good to point out that when $t=0$, equation 1 can be represented to an RC-circuit, where $G(t)$ and $S(t)$ are the voltage across the capacitor and the time-varying driving voltage respectively with $\tau_{0}=R C$. Therefor, a biexponential function $S(t): e^{t / \tau_{0}} e^{t / \tau_{1}}$ would be generated by a spike in the driver at $t=0$. Thus, the signal $G(t)$ is defined as the convolution of the driver function $S(t)$ with a biexponential function. Well isolated peaks can be obtained by deconvolving 
the signal $G(t)$ to find the driver function $S(t)$. The numerical steps that should be done are as follow:

- deconvolving the signal of skin conductance time-series in order to find the driver function;

- isolating single peaks in the driver function;

- reconstructing the individual SCRs by convolving the peaks identified in the driver function.

For simplicity in the numerical analysis, it is convenient to work with two first order equations instead of the second order differential equation 1 :

$$
h(t)=\tau_{1} \frac{d G}{d t}+G(t)
$$

and

$$
S(t)=\tau_{0} \frac{d h}{d t}+h(t)
$$

The raw signal is an approximation of the continuous curve $G(t)$ sampled at $1 \mathrm{~ms}$, which is small compared with the time scale of SCRs:

$$
G_{i}=G(i \triangle t)
$$

The discrete values for $d G / d t$ can be found by forward differencing:

$$
\left(\frac{d G}{d t}\right)_{i}=\frac{G_{i+1}-G_{i}}{\triangle t}
$$

We can define $h_{i}$ and $S_{i}$ too. The discretized versions of equation 2, 3 are used to calculate $h_{i}$ and $S_{i}$.

$$
\begin{gathered}
h(t)_{i}=\tau_{1}\left(\frac{G(t)_{i+1}-G(t)_{i}}{\triangle t}\right)+G(i \triangle t) \\
S(t)_{i}=\tau_{0}\left(\frac{h(t)_{i+1}-h(t)_{i}}{\triangle t}\right)+h(t)_{i}
\end{gathered}
$$

\section{EXPERIMENTS AND RESULTS}

This section reports experimental results and discusses about the analysis of the physiological data which can be very useful in decision support system for health care applications. In our first set of experiments, we evaluate the physiological data set which have been characterized as a behavioral measure of emotional reactivity. Figure 4 shows the physiological responses to stimulating sound which occurs between 20 to 30 seconds. It would allow us to properly compare all the sensors outputs for a 60 seconds window when the acoustic startle has been administered for $500 \mathrm{~ms}$ with $120 \mathrm{~dB}$.

Our feature analysis proves that there exists a correlation between the physiological data. There are several scenarios in which physiological data can be analyzed. Let us point one of the most important one, EDA. Hence, it is very important to devise effective signal processing tools for analyzing EDA signals for the suicide case. Let us spend a few words on these techniques.

Filtering of the EDA signal is performed with the goal of removing the unwanted noises. In the event that the environment influences the recording activity, noise is coupled into

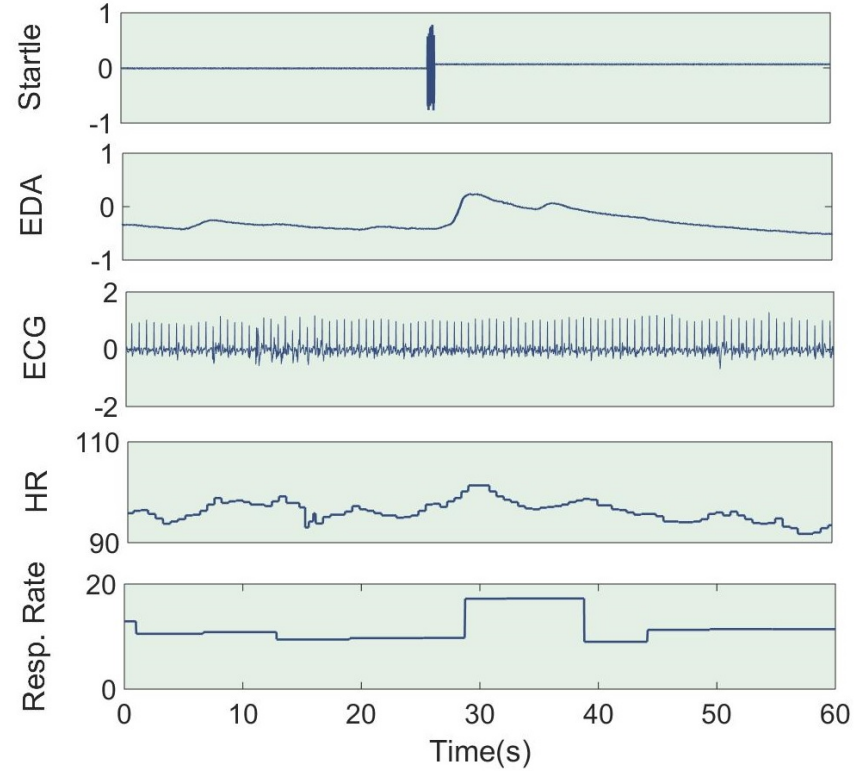

Fig. 4. The representation of acoustic startle location followed by EDA, ECG, HR and Resp. Rate signals. The changes in these signals are recognizable after the stimulus.

the EDA. To avoid unpredictable effects brought by noise, filtering becomes important for later processing. According to the spectrogram of a $30 \mathrm{~s}$ window shown in figure 5 which describes how the energy of the signal changes with big changes in the signal, the main spectrum of EDA occurs in the low frequency range with some spikes and tolerations in higher frequencies between 10 to 25 seconds. Thus, the system filters the original EDA signal using a 4 th order low-pass Butterworth filter with the cutoff frequency of $5 \mathrm{~Hz}$.

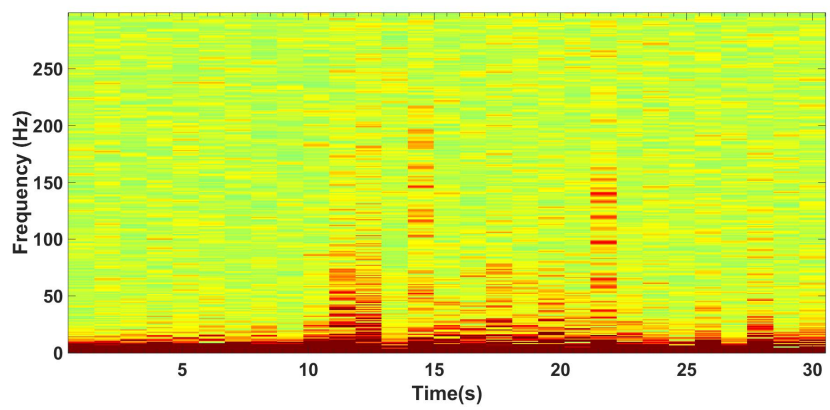

Fig. 5. A spectrogram of EDA signal.

Skin conductance is a widely used measure of psychological arousal. As it is argued in the model shown in previous section, the model is proposed for full decomposition of SC data into tonic and phasic components as:

$$
S C=S C_{\text {tonic }}+S C_{\text {phasic }}
$$

The abbreviations for phasic and tonic EDA components need to be evaluated separately. The phasic and tonic driver's changes in electrical conductivity are showed in figure 6 .

The SCR amplitude can be considered as an index of sympathetic activity. SCL is related to amplitude of the tonic 

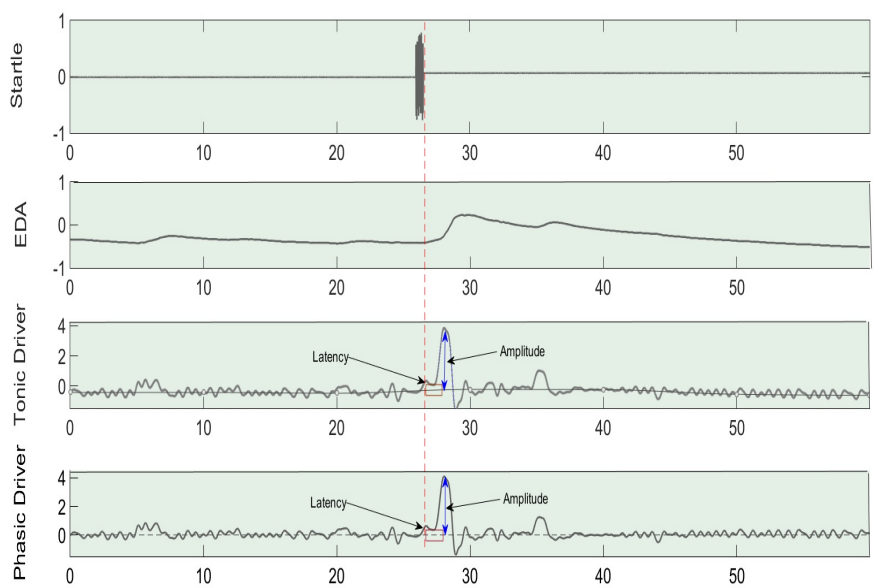

Fig. 6. Extracting tonic and phasic components from Electrodermal Activity signal.

EDA signal at the time when the stimulus is delivered. In addition, onset latency can also be measured to temporal characteristics of the SCR. As reported by [19], these SCR's temporal characteristics are not well understood as amplitude, and related to psycho-physiological processes now, but there is possibility to evaluate the SCR recovery time for applying stimulus for several times.

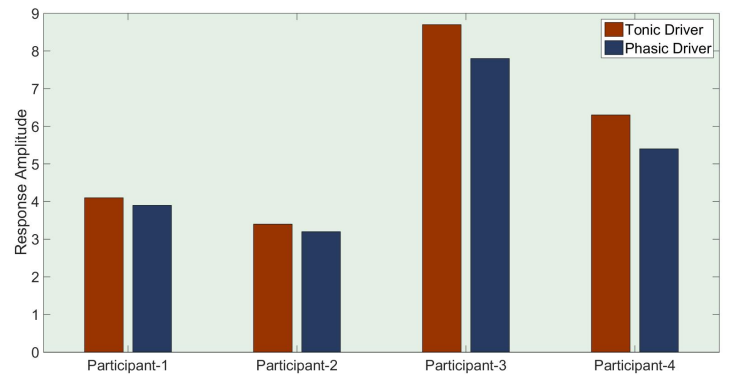

Fig. 7. Tonic and phasic response amplitudes for all participants.

Demonstrated in figure 7 is the tonic and phasic response amplitudes for all participants which could be used in evaluating SCL and SCR, respectively.

\section{CONCLusions}

The analysis of physiological data in the case of suicide risk assessment can be very useful for measuring and understanding of emotion reactivity. Hence, it is very important to devise an effective method to analyze the data when the participant faces a stimulus which in this study was an acoustic startle. This paper presents novel model in detecting the tonic and phasic components from electrodermal activity which is able to measure SCRs, its amplitude and latency. We provide physiological evidence of a reactivation for psychological stimuli through extracting tonic and phasic responses from EDA after the startle administered. This performance for promising analysis of multimodal data suspected of having a suitable well recorded dataset.
Further work is under way to improve features extraction for design an intelligent wearable system to detect and alarm for psychophysiological's changes in daily life. The proposed techniques are intended a high-volume accuracy to detect the emotional reactivity. Furthermore, this study can be designed as a detector which can be implemented for real-time detection either in software or embedded in hardware and installed in android devices for health care applications. Nonetheless, the detection of the emotion reactivity for response to startle is the first step of this research. The software system depicted in this work can be considered as a decision support system in health care center.

\section{REFERENCES}

[1] "Facts and figures. (n.d.). retrieved april 6, 2015, from." https://www. afsp.org/understanding-suicide/facts-and-figures.

[2] "National suicide data \& statistics. (n.d.). retrieved in 2013, from." http: //www.cdc.gov/violenceprevention/suicide/statistics/index.html.

[3] A. E. Crosby, L. Ortega, and C. Melanson, "Self-directed violence surveillance: Uniform definitions and recommended data elements," Atlanta, GA: Centers for Disease Control and Prevention, 2011.

[4] C. Perlman, E. Neufeld, L. Martin, M. Goy, and J. Hirdes, "Suicide risk assessment inventory: A resource guide for canadian health care organizations," Toronto, ON: Ontario Hospital Association and Canadian Patient Safety Institute [Internet], 2011.

[5] D. Wasserman, Z. Rihmer, D. Rujescu, M. Sarchiapone, M. Sokolowski, D. Titelman, G. Zalsman, Z. Zemishlany, and V. Carli, "The european psychiatric association (epa) guidance on suicide treatment and prevention," European psychiatry, vol. 27, no. 2, pp. 129-141, 2012.

[6] M. Gliatto and A. K. Rai, "Evaluation and treatment of patients with suicidal ideation," American family physician, vol. 59, pp. 1500-1513, 1999.

[7] R. I. Simon, Concise guide to psychiatry and law for clinicians. Amer Psychiatric Pub Incorporated, 2001.

[8] E. J. Stanford, R. R. Goetz, and J. D. Bloom, "The no harm contract in the emergency assessment of suicidal risk.," Journal of Clinical Psychiatry, 1994.

[9] K. M. Malone, "Pharmacotherapy of affectively ill suicidal patients," Psychiatric Clinics of North America, vol. 20, no. 3, pp. 613-624, 1997.

[10] J. Schumm, M. Bachlin, C. Setz, B. Arnrich, D. Roggen, and G. Troster, "Effect of movements on the electrodermal response after a startle event," in Pervasive Computing Technologies for Healthcare, 2008. PervasiveHealth 2008. Second International Conference on, pp. 315 318, IEEE, 2008.

[11] P. Wang, L. A. Baker, Y. Gao, A. Raine, and D. I. Lozano, "Psychopathic traits and physiological responses to aversive stimuli in children aged 911 years," Journal of abnormal child psychology, vol. 40, no. 5, pp. 759769, 2012.

[12] W. Boucsein, Electrodermal activity. Springer Science \& Business Media, 2012.

[13] J. Malmivuo and R. Plonsey, Bioelectromagnetism: principles and applications of bioelectric and biomagnetic fields. Oxford university press, 1995.

[14] M. Benedek and C. Kaernbach, "A continuous measure of phasic electrodermal activity," Journal of neuroscience methods, vol. 190, no. 1, pp. 80-91, 2010.

[15] D. Alexander, C. Trengove, P. Johnston, T. Cooper, J. August, and E. Gordon, "Separating individual skin conductance responses in a short interstimulus-interval paradigm," Journal of neuroscience methods, vol. 146, no. 1, pp. 116-123, 2005.

[16] L. E. Nacke, M. Kalyn, C. Lough, and R. L. Mandryk, "Biofeedback game design: using direct and indirect physiological control to enhance game interaction," in Proceedings of the SIGCHI conference on human factors in computing systems, pp. 103-112, ACM, 2011.

[17] D. R. Bach, J. Daunizeau, N. Kuelzow, K. J. Friston, and R. J. Dolan, "Dynamic causal modeling of spontaneous fluctuations in skin conductance," Psychophysiology, vol. 48, no. 2, pp. 252-257, 2011.

[18] V. G. Macefield and B. G. Wallin, "The discharge behaviour of single sympathetic neurones supplying human sweat glands," Journal of the autonomic nervous system, vol. 61, no. 3, pp. 277-286, 1996.

[19] J. T. Cacioppo, L. G. Tassinary, and G. Berntson, Handbook of psychophysiology. Cambridge University Press, 2007. 